\title{
An empiricist guide to animal personality variation in ecology and evolution
}

\author{
Sasha R. X. Dall ${ }^{1 *}$ and Simon C. Griffith ${ }^{2}$ \\ Centre for Ecology and Conservation, Biosciences, College of Life and Environmental Sciences, University of Exeter, Penryn, Exeter, UK \\ 2 Department of Biological Sciences, Macquarie University, Sydney, NSW, Australia
}

\section{Edited by:}

Claus Wedekind, University of

Lausanne, Switzerland

\section{Reviewed by:}

Laura J. May-Collado, University of

Puerto Rico, Puerto Rico

Gonçalo C. Cardoso, Universidade

do Porto, Portugal

*Correspondence:

Sasha R. X. Dall, Centre for Ecology and Conservation, Biosciences,

College of Life and Environmental Sciences, University of Exeter,

Penryn Campus, Penryn TR10 9EZ,

Exeter, UK

e-mail: s.r.x.dall@exeter.ac.uk
The study of animal personality variation promises to provide significant new insight into the way that behavior evolves in animals, along with its ecological and evolutionary influences. We strongly advocate more empirical work in this exciting and rapidly expanding research area, but hope that new studies adopt a more hypothesis-driven and/or experimental approach than seems to be usual at the moment. Here we outline what we feel is "good practice" to the many empiricists that are keen on pursuing work in this field. We highlight the substantial body of theoretical work that exists for providing well-reasoned hypotheses, which new empirical studies should be designed to test. Furthermore, using a brief review of existing work on the behavioral ecology of animal personality variation in the zebra finch-one of the more widely used model systems in this field-we stress the importance of understanding the ecology of the chosen study animal, and the problems that are likely to arise by neglecting to identify or account for the structure of behavioral variation that is often likely to occur.

Keywords: animal personality, theoretical ecology, experimental design, behavioral ecology, zebra finch
The study of animal personality variation has been the focus of a burgeoning research effort over the past decade, with the realization that the consistent individual differences in behavior that characterize it are the target of different forms of selection (Dingemanse and Wolf, 2010; Schuett et al., 2010; Wolf and Weissing, 2010), and can be substantial drivers of a range of important ecological and evolutionary processes (Sih et al., 2012; Wolf and Weissing, 2012). In keeping with the broad consensus in the evolutionary and behavioral ecology literature, we use the term personality variation to refer here to the existence of substantial variation in behavior among individuals within a population, with at least some individuals showing behavior that is consistent (i.e., individual differences remain stable) across time and/or contexts. Empirical studies of animal personality in the context of ecology and evolution, have been influenced by the long-established body of research conducted in the fields of animal and human psychology that is typically focused on characterizing inter-individual variation in behavior from ratings (by either the focal subjects or expert observers of focal subjects) derived from questionnaires (Gosling, 2001).

An accessible way of entering this new and exciting field of research has been to adopt standardized tools for measuring individual variation in response to controlled environments and stimuli. It has been advocated that the widespread adoption of a very general framework and standardized methodologies such as the open-field test (where individual behavior is assayed in unfamiliar, "open" spaces: Archer, 1973) provides a useful framework for addressing the ecology and evolution of animal personality variation (Reale et al., 2007). However, perhaps as a consequence, most of the recent empirical studies of animal personality published in the fields of evolution or ecology have been almost entirely descriptive and "question free." Despite requiring the capture and handling of animals, the assays of personality using, for example, open field tests, the presentation of novel objects, or startle responses are simply standardized tools for assaying variation, rather than being true manipulative experiments in themselves. Studies of natural variation are currently still useful in a field in which relatively few species have yet been the focus of more than a single study of personality variation. However, the interpretation of such observational findings will remain ambiguous without widespread experimental manipulations to test a priori hypotheses about the evolutionary and ecological factors underpinning behavioral variation of this type. Therefore, it is important that any observational studies that are attempted are designed with specific hypotheses in mind based on the ecology of the study species and theoretically-derived predictions (e.g., Table 1).

Given this backdrop, we would like to present a road map for those keen to enter this area of research, which will increase the insight provided by new empirical studies, and help newcomers avoid some of the pitfalls that have limited the conclusions and interpretations of many of the studies conducted to date.

The two most important principles we advocate, the reasons for which we will discuss further below, are:

1. Consider the ecology and evolutionary context of your species carefully and explore the theoretical literature from the perspective of that species. Focus on the personality variation in suites of behavioral traits likely to be most ecologically relevant to that particular species, and design assays that are best able to capture that appropriately.

2. Adopt an experimental and/or hypothesis-driven approach designed around a temporal framework that adequately 
Table 1 | Some empirical questions derived from theoretical models of the evolution and maintenance of animal personality variation (individuals that differ consistently in their behavior from one another within populations).

\section{IF DRIVEN BY RESOURCE ACOUISITION TRADE-OFFS:}

- Do more intense trade-offs between the mortality risks (e.g., predation risk) associated with resource acquisition (e.g., foraging) and/or assimilation (e.g., growth rates) result in more pronounced personality variation in behavior under risk (Mangel and Stamps, 2001; Stamps, 2007)?

- Do strong (life history) trade-offs between current and future reproduction (e.g., when reproduction is associated with lost opportunity costs to locate very high quality resources) generate pronounced boldness-aggression syndromes (Wolf et al., 2007)?

- Are consistent individual differences in the tendency to lead conspecifics both to and from cover when foraging under predation risk predicted by initial differences in energetic state (e.g., body condition) and is their persistence affected by how similar foraging experience is for the individuals involved (Rands et al., 2003; Dall et al., 2004)?

- Do individuals with higher metabolic rates show consistently lower levels of information use (Mathot and Dall, 2013), along with consistently higher foraging intensities under predation risk (Houston, 2010)?

- Is more pronounced personality variation (both within and across contexts) associated with situations where relative metabolic rates affect the ability to win fights over resources and/or foraging success on different options (Wolf and McNamara, 2012)?

\section{IF DRIVEN BY SOCIAL FEEDBACK:}

- Do more intense social interactions (e.g., longer affiliations) generate stronger personality variation, particularly in cooperative contexts (McNamara et al., 2009)?

- Does the ability to adjust decisions about how much to compete based on the competitive history of opponents (e.g., whether or not fights over access to resources are witnessed by third parties) affect how consistently individuals differ in their competitive behavior (Dall et al., 2004; Wolf et al., 2011)?

- Do the costs of switching among different options (e.g., food patches) affect the proportion of individuals in a social group that actively monitor the quality of the different options (e.g., the relative success rates of individuals on different patches) (Wolf et al., 2008)?

- Does higher uncertainty about one's own state (e.g., relative quality) and/or more costly signals generate stable individual differences in signaling strategies (both for producing and interpreting signals) and any other related behaviors (e.g., aggression levels) (Botero et al., 2010)?

- Does variation in value of coordinating group activities (e.g., when variation in predation risk affects the value of coordinated movement) drive personality variation in leadership tendencies (Johnstone and Manica, 2011)?

See text and Dingemanse and Wolf (2010) for more details.

captures the important variation in repeatability within and amongst individuals.

\section{THEORETICAL BACKGROUND AND EXPERIMENTAL TESTS}

Although the study of animal personality variation in the context of ecology and evolution is less than 10 years old, there is a relatively rich theoretical literature that has already been well reviewed (e.g., Dingemanse and Wolf, 2010; Wolf and Weissing, 2010). However, for a newcomer, an inconsistent and developing terminology can be somewhat difficult to navigate. The dearth of incisive experimental work also suggests that there is a need for some simple guidance about the approaches that should be followed in conducting good empirical work tackling a priori (theoretically-derived) predictions. There are two main branches of theory that have explored the drivers of (selection pressures on) animal personality variation, which are ready for empirical hypothesis testing at the behavioral level (see Wolf and Weissing, 2012 for a summary of theoretical predictions about the ecological and evolutionary consequences of personality variation). This work has focussed on identifying the key ecological factors that can select for both substantial inter-individual variation AND individual consistency to coevolve within populations (see Dingemanse and Wolf, 2010; Wolf and Weissing, 2010 for more detail). A number of theoretical papers have explored the role of individual life-history and metabolic trade-offs and the way in which they can drive consistent variation in suites of behaviors (e.g., Stamps, 2007; Wolf et al., 2007; Houston, 2010; Careau and Garland, 2012; Wolf and McNamara, 2012). These ideas are best summarized and encapsulated in the recent work defining
"Pace of Life Syndromes" (Reale et al., 2010), and are focussed on the role that resource-acquisition/assimilation trade offs play in driving the evolution and maintenance of personality variation.

The other important theoretical perspective is that the amongindividual variation in consistent patterns of behaving can be selected for directly in social (including sexual) contexts, where an individual's behavioral responses are determined by the interactions of other conspecifics and the consistent responses that they make (Dall et al., 2004; McNamara et al., 2009; Bergmüller and Taborsky, 2010; Schuett et al., 2010; Wolf et al., 2011). The basic idea is that, when animals interact socially, they can pay attention to each other's behavior to make better decisions. Once individuals are using such basic social information, it changes selection on how to pattern behavior over time in the presence of audiences. In some contexts (e.g., when competing aggressively for resources or interacting cooperatively) this can select for individual behavioral differentiation (Dall et al., 2004; McNamara et al., 2009; Wolf et al., 2011) or it can create negative frequency dependent selection on being adaptively flexible (Wolf et al., 2008).

Together, these non-mutually exclusive sets of theoretical work provide an excellent framework for approaching the study of variation in animal personality, although, to date, very few of the predictions that they make for understanding the variation across and within animal populations have been directly tested. Table $\mathbf{1}$ summarizes some empirical research questions derived from this theoretical work so far (see Dingemanse and Wolf, 2010; Wolf and Weissing, 2010 for more details). Trade-offs and state-dependent feedback loops have been proposed as common features driving patterns of variation within populations across both sets of 
theoretical work (Dall et al., 2004; Luttbeg and Sih, 2010), and therefore, well designed manipulations of key metabolic, lifehistory, environmental, or social variables should readily test the strength of theoretical predictions (Table 1).

The very few examples of experimental work (including "natural experiments") in this field illustrate some of the approaches that can be taken. An example of what we would consider "good practice" involved experiments on captive zebra finches that directly tested hypotheses about the role of sexual selection on personality variation (Schuett et al., 2010). Schuett et al. (2011a,b) showed that personality variation impacted both the fitness consequences of pairing up according to behavioral type (via a forced-pairing, cross-fostering experiment: Schuett et al., 2011a) and mate choice decisions (by manipulating the apparent behavioral type of males in a standardized mate choice protocol: Schuett et al., 2011b) in captive zebra finch populations. There are a number of other studies that similarly manipulate personality variation to assess its impact on measures of fitness (e.g., Sih and Watters, 2005; Riebli et al., 2012; Sweeney et al., 2013). Most impressive, though, are the very few studies that have isolated the effects of specific factors that were hypothesized a priori to influence the structure of personality variation within populations. These include a comparison across multiple populations that has taken the "natural experiment" approach to investigate a purported relationship between predation or parasite intensity and the distribution of personality variation in three-spined sticklebacks (Dingemanse et al., 2007). Moreover, Bell and Sih (2007) went on to manipulate predation intensity on captive stickleback populations and demonstrated the hypothesized (causal) effect of predation intensity on selection for boldness-aggression syndromes. Finally, Laskowski and Bell (2013) manipulated intraspecific competition over access to food in captive sticklebacks and corroborated theoreticallyderived predictions (Table 1) that heightened competition levels should promote stronger personality variation.

\section{THE IMPORTANCE OF MEASURING BEHAVIOR REPEATEDLY}

A pretty fundamental problem facing researchers interested in studying animal personality variation is that, as humans, we effortlessly cue in on individuality and individual patterns behavior. Perhaps as a consequence, we have been too ready to dismiss the evolutionary importance of such behavior (e.g., its functional significance in non-human animals) and so human-centerd (psychological) definitions of personality range from the vaguely broad: "individual differences within species" (Gosling and John, 1999), to the idiosyncratically human-specific: "those characteristics of individuals that describe and account for consistent patterns of feeling, thinking and behaving" (Pervin and John, 1997). Indeed, both extremes are of limited value when investigating measurable patterns of individual variation in an evolutionary and ecological context (e.g., across species). Clearly further elucidation is needed. There are two distinct components that must be included within the scope of animal personality variation from an evolutionarily and ecologically functional standpoint (see Stamps and Groothuis, 2010; Kight et al., 2013 for a more exhaustive discussions of the issue). First and foremost, investigators must consider behavioral variation amongst individuals within otherwise homogenous populations or groups of the same species when they study animal personality. Crucially, however, researchers must simultaneously consider the patterns of behavior that individuals exhibit over some portion of their lifetimes and/or across the different biological contexts they face (e.g., foraging, mating, socializing etc.), since a hallmark of personality is the relative stability or consistency of individual behavior over some portion of a life history. Indeed, the vast majority of existing studies on animal personality variation fall within this scope. Moreover, by adopting this stance, we do not restrict ourselves to studying inter individual variation that is generated by any specific proximate mechanisms (e.g., coded for genetically), thus avoiding potential confusion over levels of explanation (Tinbergen, 1963) by focusing on observable patterns of behavior per se. In this way, animal personality studies can encompass variation that is genetically driven, while also considering inter individual variation that involves substantial behavioral plasticity, need not be stable for a lifetime and depends on social status or condition.

Given this emphasis on patterns of variation and consistency, it is necessary to structure measurements of behavior in such a way to be able to quantify this class of variation at biologically significant scales for the species in question. The basic approach must therefore involve taking repeated measurements of behavior from the same individuals across multiple ecologically important time periods and/or contexts (e.g., within vs. among breeding seasons; while breeding vs. not breeding etc.: see Bell, 2012 for more details). It is also important to ensure that you get estimates of behavioral repeatabilities (i.e., you get repeated measures) for ALL of the individuals in your study population. This is because theory suggests that individual differences in the consistency or flexibility of behavior can evolve in certain contexts (e.g., Wolf et al., 2008). Therefore a single population-representative repeatability (e.g., mean repeatability) may not be biologically meaningful and quantifying the full-range of individual-level patterns of behavioral variation will be necessary to critically test theoretical predictions. Dingemanse et al. (2010) have described the 'reaction norm' approach that provides an excellent framework for structuring and analysing data sets in a way that will capture the variation within and between individuals in behavioral traits over time and across different ecological, or life-history contexts. As such it relies heavily on mixed-effect statistical modeling approaches (Dingemanse and Dochtermann, 2013). Researchers that follow the detailed recommendations made in these reviews will maximize their abilities to fully-quantify personality variation within their study populations, if it is present.

\section{A SPECIES-SPECIFIC APPROACH TO THE STUDY OF VARIATION IN PERSONALITY}

As in all other areas of biology, the starting point for good research should be the choice of a species that is appropriate for the particular question that you wish to address. There is nothing inherently wrong with studying systems that are readily accessible, and it is perhaps no surprise, for instance, that more research has been conducted on existing research populations of tits (Cyanistes caeruleus or Parus major), or the domesticated zebra finch (Taeniopygia guttata), than other avian species. However, to date, most of the work on these species has focused on demonstrating the existence of personality, methods for measuring 
personality (Herborn et al., 2010; Kluen et al., 2012), and correlates of particular personality traits that have been explored in the absence of clear a priori theoretically driven predictions (e.g., David et al., 2012; Patrick et al., 2012), with highly variable methodologies and sample sizes (Garamszegi et al., 2012).

The difficulty of non-manipulative studies (or observational studies that are not designed to test a specific hypothesis) is that the interpretation of results can be very difficult, if not impossible in the absence of a proper understanding of a species. For example, one of the more widely used assay of personality variation is the "candidate behavior" approach (Reale et al., 2007), which focuses on assay-derived categories of behavior that are difficult to interpret and define across and within populations. A relevant example here is to consider the open field test (Archer, 1973), in which an animal is released into an unfamiliar cage/ box and the degree of movement in a set period of time is assessed (e.g., Dingemanse et al., 2004; Schuett and Dall, 2009). Many researchers interpret this test as assaying "boldness" or exploratory behavior. However, this may not be the correct interpretation for species that have evolved with ambush predators (i.e., take refuge in open areas) and forage under cover, or are highly social (Carter et al., 2013). Indeed, Carter et al. (2013) detail the problems that arise from the widespread use of such standardized "personality assays" without careful consideration of how well they assay what you think they assay in your study species, and the ecological relevance of any given assay for different animal systems. We agree with many of their recommendations to deal with the first issue, especially that multiple tests should be designed for each functional category of behavior that has been identified a priori as being of interest, and that individual tests should be validated against each other to ensure personality variation within the functional domain of interest can be reliably quantified. However, we differ somewhat in our recommendations about how researchers should design assays in the first place. As we have stated all along, we feel that, as with any study of animal behavior from an ecological or evolutionary perspective, assays should be designed to capture variation in behavioral responses to key ecological trade-offs that have been identified a priori as likely influencing animal personality variation. So, for instance, if you are attempting to test the hypothesis that strong trade-offs between mortality and resource acquisition are likely to favor individual differences in attitudes to risk while foraging (Table 1), you should design (or customize) assays to probe, in a standardized way, the willingness to look for food in situations that are perceived as posing a significant risk of mortality for your focal species.

In addition to the issue of reliably assaying behavior in an ecologically meaningful manner, it is also important to design personality testing to capture hidden structure to variation measured along a continuum that can be predicted theoretically. For instance, in open field tests it is typically assumed by many of the empirical studies that individual variation will be distributed along a continuum from individuals that move around lots, and are "exploratory" to those that are "non-exploratory" and essentially don't move around. It is often believed, for example, that this exploratory aspect of personality will be related to dispersal ability and inversely related to the risk of predation that an individual exposes itself to. However, theoretical work often predicts that the variation in stable individual behavior across a population will not be so continuous, and this can start to make clear interpretations very difficult. For example, in their recent study of the great tit, Titulaer et al. (2012) found that the relationship between exploratory behavior and flexible learning abilities was discontinuous across the population: it manifest in opposite directions in males and females. In this study, the sex-specific personality variation was relatively easy to identify and account for in both experimental design and analyses, because sex was easy to assign and is an obvious feature of individuals to record in any study of behavior. However, a more interesting, and problematic situation arises where we have a similar degree of structuring amongst the individuals within a population that is not so readily identified, or accounted for. Behavioral polymorphisms can often be very cryptic (driven by hidden "state variables" like reputation or energy levels), and indeed many of the theoretical models have predicted the emergence of multiple stable personalities (e.g., Wolf et al., 2007). Where such polymorphism occurs within populations, we would not necessarily expect a simple linear or continuous pattern of variation, and this makes interpretation of datasets very difficult without having assayed the relevant state-variables from the outset of data collection. For example, if individuals within a population follow different strategies then the relationship between a particular behavioral type or syndrome and other variables (e.g., lifetime reproductive success) is likely to vary dependent upon the polymorphism. Counter-acting relationships exhibited in different morphs or subsets of a population would make it very difficult to determine or examine relationships in a population-wide sample in the absence of a structured data collection protocol designed a priori to assign individuals to their biologically-relevant categories.

\section{PERSONALITY VARIATION OF A MODEL SYSTEM IN AN ECOLOGICAL CONTEXT}

To help illustrate the pitfalls we have highlighted so far, along with some of the approaches we are advocating as "good practice," we now provide a brief review of the behavioral ecology of zebra finches from an animal personality perspective. Although studies of the zebra finch have made a disproportionate contribution to a number of fields in animal behavior and evolutionary biology (Griffith and Buchanan, 2010), and have already been the focus of around 15 publications relating to variation in personality in captive populations, we are only recently developing an understanding of the ecology of wild populations. There are a number of aspects of the life-history and behavior of the wild zebra finch that have the potential to influence the way that studies of personality variation in this species are approached and interpreted. A brief review of some of these issues, for a bird that has already been the focus of more empirical work than almost any other single species in the field of animal personality variation, should help to focus attention on the importance of a careful consideration of species ecology and behavior for future studies in any species from this perspective. The contrast between recent work on personality in this captive model species (largely conducted on individually assayed zebra finches in a relatively simple and predator-free captive environment), with the complex social ecology of the wild bird highlights issues that are likely to be common to many species that have been, or will become, the focus of 
further work in this area. Whilst the differences will not perhaps as extreme as those between captive domesticated zebra finches and their wild free-living counterparts, it is still very easy to lose sight of the way that free-living individuals operate, when we are capturing them and assaying them in isolation. Specifically we identify two important considerations in the extent to which individuals operate on their own or in a social context, and the pattern of variation in behavioral traits across a population.

\section{THE EFFECT OF SOCIAL ISOLATION}

In the wild, zebra finches pair for life (probably shortly after reaching sexual maturity) and are almost always in close proximity to their partner, both during reproductive cycles and throughout non-breeding periods (Mariette and Griffith, 2012a), and have a very low level of infidelity for a passerine (Griffith et al., 2010). One of the few periods when the pair is out of immediate contact with one another is during the incubation period (when the male and female take turns to separately incubate the eggs). Pairs perform regular and characteristic acoustic duetting behavior (Elie et al., 2010), and become physiologically stressed when socially isolated, moderating their vocalizations accordingly (Perez et al., 2012). Above the level of the pair bond, zebra finches will often form associations with other pairs and coordinate their reproductive activity in small colonies with between 1 and 10 other pairs in close proximity (Mariette and Griffith, 2012b). Given the highly social nature of the wild zebra finch, it is not clear what persistent effects might be caused on domesticated zebra finches by keeping them, or assaying personality variation in social isolation. However, it is interesting that in their study Mainwaring et al. (2011), found birds to have a reduced level of exploratory behavior in social groups than when assayed in isolation. It will be interesting to follow up on these observational findings to test whether, for example this difference may have been driven be anxiety in isolated birds, or the social feedback dynamics that occur in small groups.

The strong pair bond of the wild zebra finch and the level of synchronized behavior between the pair (Mariette and Griffith, 2012a), also help to place the findings of Schuett and Dall (2009) in a better context. In their study they found that the exploratory behavior of individuals became more similar (coordinated) when birds foraged together in opposite sex pairs (compared to when such behavior was assayed repeatedly on isolated individuals), and this does make sense in a species in which behavior is so tightly integrated between a male and female.

Typically, candidate behavior tests are conducted on individuals held in unfamiliar surroundings and isolated from other conspecifics. This approach does present some challenges for species such as the zebra finch in which individuals are rarely alone, or even more social species such as cooperatively breeding species, that also are rarely alone and have highly coordinated group behavior on a daily basis in, or out of the breeding season (e.g., Sorato et al., 2012). Further work is required to determine the effect of social isolation, or isolation from strongly affiliated individuals, on personality assays and whether this might be introducing persistent or non-random biases across populations. Future studies should certainly consider such effects when designing assays and experimental procedures.
A likely way forward here would be to use ongoing behavioral assays (perhaps conducted on free-living animals: e.g., behavior on artificial food patches), which could be conducted on individuals across a variety of social contexts, to be analyzed with a mixed model approach that would enable researchers to partition out the effects of social context on the repeatability of behavior (e.g., Nakagawa and Schielzeth, 2010). For example, to ask questions such as how does an individual's pattern of exploratory behavior change when it is: (a) with its sexual partner; (b) amongst a group of familiar conspecifics; (c) unfamiliar conspecifics; (d) a potential new sexual partner?

\section{BEHAVIORAL AND GENETIC STRUCTURING WITHIN POPULATIONS}

Zebra finches are opportunistic breeders in the wild and individuals can breed over extended periods of the year, and at a variety of different breeding densities (Zann, 1996). Recent work has identified two strategies within wild populations, with many birds coordinating and aggregating their reproductive attempts both temporally and spatially with a number of con-specifics in high density colonies, but a notable proportion of the same population choosing to nest singly in very isolated patches (Mariette and Griffith, 2012b). Pairs nesting in solitary nests were better at finding experimental foraging patches, and were better at adjusting parental care to experimentally enlarged broods than birds following a more colonial nesting strategy (Mariette and Griffith, 2013). The extent to which these different reproductive strategies are state-dependent (e.g., dependent on condition when first encountering a suitable breeding habitat) is currently unclear however there are no obvious genetic or morphological predictors of the strategy that an individual will follow. These colonial and solitary birds appear to have different information about the environment, and different patterns of sociality and foraging behavior, which are likely to expose them to different risks and adaptive trade-offs. For instance, if high density aggregations of breeding finches attract the nomadic, and opportunistic raptors that occur in the Australian arid zone (Marchant and Higgins, 1993), solitary breeders may experience lower levels of predation risk, which may encourage higher rates of resource acquisition and better information about local resources. It is difficult to identify whether an individual is a solitary or colonial bird by morphological or molecular markers and therefore it is very difficult to account for, or explore the relationship between this behavioral strategy and personality variation, even though we might anticipate some dependencies. For example, colonial birds might be expected to be highly socially tolerant and be less likely to explore novel habitats asocially, while solitary birds are likely to show lower levels of socialization, be more aggressive, value asocial information more etc.

Furthermore, developing an understanding of these two strategies and how they operate in wild birds can completely switch around the interpretation of observed patterns of personality variation. For example, most of the work on personality variation in captive zebra finches has interpreted variation in performance on open field tests (novel environment assays) as reflecting individual differences in the tendency to take risks: i.e., individual differences in the fitness value of taking a gamble on potential novel opportunities in unfamiliar environments (e.g., Schuett and 
Dall, 2009; Schuett et al., 2011a,b). However, recognizing that there are these two different lifestyles in wild zebra finches suggests that a more ecologically realistic interpretation might be that "highly exploratory" individuals (i.e., those that move around a novel environment more when alone) are those individuals that perceive the world as less risky (whose prior expectation of risk is lower) and therefore place higher value on local information about resources etc. (Dall, 2010). By contrast, highly social individuals may not rely on their own acquired knowledge of an environment and be more dependent on the acquisition of social information to identify current ecological opportunities. The challenge here is how we can interpret our results on personality in the absence of such ecological knowledge. For example an individual might be a very poor explorer in it's own right and have a very poor skill set at finding food alone in the environment. However if such low explorers are highly sensitive to social cues, and only ever operate as part of a larger social group then their poor exploratory personality is not necessarily the fitness disadvantage we might initially expect. We need to understand the extent to which individuals operate alone or as part of a larger social structure, and integrate that knowledge as part of the study of animal personality variation. We need to understand how reliant individuals are on each other, and how that relates to personality traits with respect to other aspects of ecology and behavior. There is lots of interesting variation in the degree of individual sociality across and within species with some excellent recent breakthroughs in understanding the neuronal and molecular basis for some of that variation (e.g., Goodson et al., 2009; Goodson and Kingsbury, 2011). Furthermore, the timely emergence of interest in the analyses of animal social networks (Krause et al., 2009), will allow future studies to integrate the study of personality variation with the social context, or strategies that different individuals within populations take. Such integrative studies will provide excellent opportunities to test some of the ideas proposed by the theoretical literature focused on the effects of social interactions on personality variation (e.g., Dall et al., 2004; McNamara et al., 2009; Schuett et al., 2010; Wolf et al., 2011).

\section{CONCLUSIONS}

It is of paramount importance to properly understand the species with which you are working before designing a study of personality variation from an evolutionary or behavioral ecology perspective. Personality variation is likely to be there, but it's very easy to overlook if you don't measure it correctly, or are unable to account for behavioral polymorphisms within your species. These might be driven by sex-, or phenotype dependent variation, that may be easy to evaluate, or they might result from cryptic behavioral polymorphisms that are very difficult to identify without a reasonably good understanding of species ecology and evolution. This is a very good reason to follow the approach we advocate here (explicitly based on ecology context), and work with species for which we have a reasonable understanding of basic ecology and behavior, and to think about how we might expect that to affect selection on patterns of personality variation.

\section{REFERENCES}

Archer, J. (1973). Tests for emotionality in rats and mice: a review. Anim. Behav. 21, 205-235. doi: 10.1016/S0003-3472(73)80065-X
Bell, A. (2012). Randomized or fixed order for studies of behavioral syndromes? Behav. Ecol. 24, 16-20. doi: 10.1093/beheco/ars148

Bell, A. M., and Sih, A. (2007). Exposure to predation generates personality in threespined sticklebacks (Gasterosteus aculeatus). Ecol. Lett. 10, 828-834. doi: 10.1111/j.1461-0248.2007.01081.x

Bergmüller, R., and Taborsky, M. (2010). Animal personality due to social niche specialisation. Trends Ecol. Evol. 25, 504-511. doi: 10.1016/j.tree.2010.06.012

Botero, C. A., Pen, I., Komdeur, J., and Weissing, F. J. (2010). The evolution of individual variation in communication strategies. Evolution 64, 3123-3133. doi: 10.1111/j.1558-5646.2010.01065.x

Careau, V., and Garland, T. J. (2012). Performance, personality, and energetics: correlation, causation, and mechanism. Physiol. Biochem. Zool. 85, 543-571. doi: $10.1086 / 666970$

Carter, A. J., Feeney, W. E., Marshall, H. H., Cowlishaw, G., and Heinsohn, R. (2013). Animal personality: what are behavioural ecologists measuring? Biol. Rev. 88, 465-475. doi: 10.1111/brv.12007

Dall, S. R. X. (2010). "Managing risk: the perils of uncertainty," in Evolutionary Behavioral Ecology, ed D. F. Westneat and C. W. Fox (New York, NY: Oxford University Press), 194-206.

Dall, S. R. X., Houston, A. I., and McNamara, J. M. (2004). The behavioural ecology of personality: consistent individual differences from an adaptive perspective. Ecol. Lett. 7, 734-739. doi: 10.1111/j.1461-0248.2004.00618.x

David, M., Auclair, Y., Giraldeau, L.-A., and Cézilly, F. (2012). Personality and body condition have additive effects on motivation to feed in Zebra Finches Taeniopygia guttata. Ibis 154, 372-378. doi: 10.1111/j.1474-919X.2012. 01216.x

Dingemanse, N. J., Both, C., Drent, P. J., and Tinbergen, J. M. (2004). Fitness consequences of avian personalities in a fluctuating environment. Proc. R. Soc. Lond. B Biol. Sci. 271, 847-852. doi: 10.1098/rspb.2004.2680

Dingemanse, N. J., and Dochtermann, N. A. (2013). Quantifying individual variation in behaviour: mixed-effect modelling approaches. J. Anim. Ecol. 82, 39-54. doi: 10.1111/1365-2656.12013

Dingemanse, N. J., Kazem, A. J. N., Reale, D., and Wright, J. (2010). Behavioural reaction norms: animal personality meets individual plasticity. Trends Ecol. Evol. 25, 81-89. doi: 10.1016/j.tree.2009.07.013

Dingemanse, N. J., and Wolf, M. (2010). Recent models for adaptive personality differences: a review. Philos. Trans. R. Soc. B Biol. Sci. 365, 3947-3958. doi: 10.1098/rstb.2010.0221

Dingemanse, N. J., Wright, J., Kazem, A. J. N., Thomas, D. K., Hickling, R., and Dawnay, N. (2007). Behavioural syndromes differ predictably between 12 populations of three-spined stickleback. J. Anim. Ecol. 76, 1128-1138. doi: 10.1111/j.1365-2656.2007.01284.x

Elie, J. E., Mariette, M. M., Soula, H. A., Griffith, S. C., Mathevon, N., and Vignal, C. (2010). Vocal communication at the nest between mates in wild zebra finches: a private vocal duet? Anim. Behav. 80, 597-605. doi: 10.1016/j.anbehav.2010.06.003

Garamszegi, L. Z., Marko, G., and Herczeg, G. (2012). A meta-analysis of correlated behaviours with implications for behavioural syndromes: mean effect size, publication bias, phylogenetic effects and the role of mediator variables. Evol. Ecol. 26, 1213-1235. doi: 10.1007/s10682-012-9589-8

Goodson, J. L., and Kingsbury, M. A. (2011). Nonapeptides and the evolution of social group sizes in birds. Front. Neuroanat. 5:13. doi: 10.3389/fnana.2011.00013

Goodson, J. L., Schrock S. E., Klatt, J. D., Kabelik, D., and Kingsbury, M. A. (2009). Mesotocin and nonapeptide receptors promote estrildid flocking behavior. Science 325, 862-866. doi: 10.1126/science.1174929

Gosling, S. D. (2001). From mice to men: what can we learn about personality from animal research? Psychol. Bull. 127, 45-86. doi: 10.1037/0033-2909.127.1.45

Gosling, S. D., and John, O. P. (1999). Personality dimensions in non-human animals: a cross-species review. Curr. Dir. Psychol. Sci. 8, 69-75. doi: 10.1111/14678721.00017

Griffith, S. C., and Buchanan, K. L. (2010). The Zebra Finch: the ultimate Australian supermodel. Emu 110, v. doi: 10.1071/MUv110n3_ED

Griffith, S. C., Holleley, C. E., Mariette, M. M., Pryke, S. R., and Svedin, N. (2010). Low level of extrapair parentage in wild zebra finches. Anim. Behav. 79, 261-264. doi: 10.1016/j.anbehav.2009.11.031

Herborn, K. A., Macleod, R., Miles, W. T. S., Schofield, A. N. B., Alexander, L., and Arnold, K. E. (2010). Personality in captivity reflects personality in the wild. Anim. Behav. 79, 835-843. doi: 10.1016/j.anbehav.2009.12.026 
Houston, A. I. (2010). Evolutionary models of metabolism, behaviour and personality. Philos. Trans. R. Soc. B Biol. Sci. 365, 3969-3975. doi: 10.1098/rstb.2010. 0161

Johnstone, R. A., and Manica, A. (2011). Evolution of personality differences in leadership. Proc. Natl. Acad. Sci. U.S.A. 108, 8373-8378. doi: 10.1073/pnas. 1102191108

Kight, C. R., David, M., and Dall, S. R. X. (2013). "The evolution of animal personality variation," in eLS (John Wiley and Sons, Ltd.). doi: 10.1002/9780470015902.a0024662. Available online at: http://onlinelibrary. wiley.com/doi/10.1002/9780470015902.a0024662/abstract;jsessionid=FED389A C4D1614382DE4E37CC8A7F5DE.f01t01

Kluen, E., Kuhn, S., Kempenaers, B., and Brommer, J. E. (2012). A simple cage test captures intrinsic differences in aspects of personality across individuals in a passerine bird. Anim. Behav. 84, 279-287. doi: 10.1016/j.anbehav.2012. 04.022

Krause, J., Lusseau, D., and James, R. (2009). Animal social networks: an introduction. Behav. Ecol. Sociobiol. 63, 967-973. doi: 10.1007/s00265-009-0747-0

Laskowski, K. L., and Bell, A. M. (2013). Competition avoidance drives individual differences in response to a changing food resource in sticklebacks. Ecol. Lett. 16, 746-753. doi: 10.1111/ele.12105

Luttbeg, B., and Sih, A. (2010). Risk, resources and state-dependent adaptive behavioural syndromes. Philos. Trans. R. Soc. B Biol. Sci. 365, 3977-3990. doi: 10.1098/rstb.2010.0207

Mainwaring, M. C., Beal, J. L., and Hartley, I. R. (2011). Zebra finches are bolder in an asocial, rather than social, context. Behav. Process. 87, 171-5. doi: 10.1016/j.beproc.2011.03.005

Mangel, M., and Stamps, J. (2001). Trade-offs between growth and mortality and the maintenance of individual variation in growth. Evol. Ecol. Res. 3, 583-593.

Marchant, S., and Higgins, P. J. (1993). Handbook of Australian, New Zealand and Antarctic Birds, Vol. 2. Raptors to Lapwings (Melbourne, VIC: Oxford University Press).

Mariette, M. M., and Griffith, S. C. (2012a). Nest visit synchrony is high and correlates with reproductive success in the wild Zebra finch Taeniopygia guttata. J. Avian Biol. 43, 131-140. doi: 10.1111/j.1600-048X.2012.05555.x

Mariette, M. M., and Griffith, S. C.(2012b). Conspecific attraction and nest site selection in a nomadic species, the zebra finch. Oikos 121, 823-834. doi: 10.1111/j.1600-0706.2011.20014.x

Mariette, M. M., and Griffith, S. C. (2013). Does coloniality improve foraging efficiency and nestling provisioning? A field experiment in the wild zebra finch. Ecology 94, 325-335. doi: 10.1890/12-0572.1

Mathot, K. J., and Dall, S. R. X. (2013). Metabolic rates can drive individual differences in information and insurance use under the risk of starvation. Am. Nat. 182, 611-620. doi: 10.1086/673300

McNamara, J. M., Stephens, P. A., Dall, S. R. X., and Houston, A. I. (2009). Evolution of trust and trustworthiness: social awareness favours personality differences. Proc. R. Soc. B Biol. Sci. 276, 605-613. doi: 10.1098/rspb.2008.1182

Nakagawa, S., and Schielzeth, H. (2010). Repeatability for Gaussian and nonGaussian data: a practical guide for biologists. Biol. Rev. 85, 935-956. doi: 10.1111/j.1469-185X.2010.00141.x

Patrick, S. C., Chapman, J. R., Dugdale, H. L., Quinn, J. L., and Sheldon, B. C. (2012). Promiscuity, paternity and personality in the great tit. Proc. Biol. Sci. R. Soc. 279, 1724-1730. doi: 10.1098/rspb.2011.1820

Perez, E. C., Elie, J. E., Soulage, C. O., Soula, H. A., Mathevon, N., and Vignal, C. (2012). The acoustic expression of stress in a songbird: does corticosterone drive isolation-induced modifications of zebra finch calls? Horm. Behav. 61, 573-581. doi: 10.1016/j.yhbeh.2012.02.004

Pervin, L. A., and John, O. P. (1997). Personality: Theory and Research. New York, NY: Guildford Press.

Rands, S. A., Cowlishaw, G., Pettifor, R. A., Rowcliffe, J. M., and Johnstone, R. A. (2003). Spontaneous emergence of leaders and followers in foraging pairs. Nature 423, 432-434. doi: 10.1038/nature01630

Reale, D., Garant, D., Humphries, M. M., Bergeron, P., Careau, V., and Montiglio, P. O. (2010). Personality and the emergence of the pace-of-life syndrome concept at the population level. Philos. Trans. R. Soc. Lond. B Biol. Sci. 365, 4051-4063. doi: $10.1098 /$ rstb.2010.0208

Reale, D., Reader, S. M., Sol, D., McDougall, P. T., and Dingemanse, N. J. (2007). Integrating animal temperament within ecology and evolution. Biol. Rev. 82, 291-318. doi: 10.1111/j.1469-185X.2007.00010.x

Riebli, T., Taborsky, M., Chervet, N., Apolloni, N., Zurcher, Y., and Hek, D. (2012). Behavioural type, status and social context affect behaviour and resource allocation in cooperatively breeding cichlids. Anim. Behav. 84, 925-936. doi: 10.1016/j.anbehav.2012.07.017

Schuett, W., and Dall, S. R. X. (2009). Sex differences, social context and personality in zebra finches, Taeniopygia guttata. Anim. Behav. 77, 1041-1050. doi: 10.1016/j.anbehav.2008.12.024

Schuett, W., Dall, S. R. X., and Royle, N. J. (2011a). Pairs of zebra finches with similar "personalities" make better parents. Anim. Behav. 81, 609-618. doi: 10.1016/j.anbehav.2010.12.006

Schuett, W., Godin, J.-G. J., and Dall, S. R. X. (2011b). Do female zebra finches, taeniopygia guttata, choose their mates based on their "personality"? Ethology 117, 908-917. doi: 10.1111/j.1439-0310.2011.01945.x

Schuett, W., Tregenza, T., and Dall, S. R. X. (2010). Sexual selection and animal personality. Biol. Rev. 85, 217-246. doi: 10.1111/j.1469-185X.2009.00101.x

Sih, A., Cote, J., Evans, M., Fogarty, S., and Pruitt, J. (2012). Ecological implications of behavioural syndromes. Ecol. Lett. 15, 278-289. doi: 10.1111/j.14610248.2011.01731.x

Sih, A., and Watters, J. V. (2005). The mix matters: behavioural types and group dynamics in water striders. Behaviour 142, 1423. doi: $10.1163 / 156853905774539454$

Sorato, E., Gullett, P. R., Griffith, S. C., and Russell, A. F. (2012). Effects of predation risk on foraging behaviour and group size: adaptations in a social cooperative species. Anim. Behav. 84, 823-834. doi: 10.1016/j.anbehav.2012.07.003

Stamps, J. A. (2007). Growth-mortality tradeoffs and personality traits in animals. Ecol. Lett. 10, 355-363. doi: 10.1111/j.1461-0248.2007.01034.x

Stamps, J., and Groothuis, T. G. G. (2010). The development of animal personality: relevance, concepts and perspectives. Biol. Rev. 85, 301-325. doi: 10.1111/j.1469-185X.2009.00103.x

Sweeney, K., Gadd, R. D. H., Hess, Z. L., McDermott, D. R., MacDonald, L., Cotter, P., et al. (2013). Assessing the effects of rearing environment, natural selection, and developmental stage on the emergence of a behavioral syndrome. Ethology 119, 436-447. doi: 10.1111/eth.12081

Tinbergen, N. (1963). On aims and methods of ethology. Z. Tierpsychol. 20, 410-433. doi: 10.1111/j.1439-0310.1963.tb01161.x

Titulaer, M., Van Oers, K., and Naguib, M. (2012). Personality affects learning performance in difficult tasks in a sex-dependent way. Anim. Behav. 83, 723-730. doi: 10.1016/j.anbehav.2011.12.020

Wolf, M., and McNamara, J. M. (2012). On the evolution of personalities via frequency-dependent selection. Am. Nat. 179, 679-692. doi: 10.1086/665656

Wolf, M., Van Doorn, G. S., Leimar, O., and Weissing, F. J. (2007). Life-history trade-offs favour the evolution of animal personalities. Nature 447, 581-584. doi: 10.1038/nature05835

Wolf, M., Van Doorn, G. S., and Weissing, F. J. (2008). Evolutionary emergence of responsive and unresponsive personalities. Proc. Natl. Acad. Sci. U.S.A. 105, 15825-15830. doi: 10.1073/pnas.0805473105

Wolf, M., Van Doorn, G. S., and Weissing, F. J. (2011). On the coevolution of social responsiveness and behavioural consistency. Proc. R. Soc. B Biol. Sci. 278, 440-448. doi: 10.1098/rspb.2010.1051

Wolf, M., and Weissing, F. J. (2010). An explanatory framework for adaptive personality differences. Philos. Trans. R. Soc. B Biol. Sci. 365, 3959-3968. doi: 10.1098/rstb.2010.0215

Wolf, M., and Weissing, F. J. (2012). Animal personalities: consequences for ecology and evolution. Trends Ecol. Evol. 27, 452-461. doi: 10.1016/j.tree.2012.05.001

Zann, R. A. (1996). The Zebra Finch. Oxford: Oxford University Press.

Conflict of Interest Statement: The authors declare that the research was conducted in the absence of any commercial or financial relationships that could be construed as a potential conflict of interest.

Received: 29 November 2013; accepted: 28 January 2014; published online: 14 February 2014.

Citation: Dall SRX and Griffith SC (2014) An empiricist guide to animal personality variation in ecology and evolution. Front. Ecol. Evol. 2:3. doi: 10.3389/fevo.2014.00003 This article was submitted to Behavioral and Evolutionary Ecology, a section of the journal Frontiers in Ecology and Evolution.

Copyright (C) 2014 Dall and Griffith. This is an open-access article distributed under the terms of the Creative Commons Attribution License (CC BY). The use, distribution or reproduction in other forums is permitted, provided the original author(s) or licensor are credited and that the original publication in this journal is cited, in accordance with accepted academic practice. No use, distribution or reproduction is permitted which does not comply with these terms. 